Artículo de investigación.

Cómo citar: R. Cárdenas. "Repositorio de Arquitectura Empresarial del Gobierno (GEAR). Un estado abierto, soportado en tecnologías de la información y la cominicación (TIC)". Inventum, vol. 15 , no. 28 , pp. $64-75$. doi: $10.26620 /$ uniminuto. inventum.15.28.2020. 64-75

Editorial: Corporación Universitaria Minuto de Dios - UNIMINUTO.

ISSN: $1909-2520$

elSSN: $2590-8219$

Fecha de recibido: 10 de enero de 2020 Fecha de aprobado: 20 de marzo de 2020 Fecha de publicación: 30 de mayo del 2020

Conflicto de intereses: los autores han declarado que no existen intereses en competencia.
Roberto Mauricio Cárdenas Cárdenas roberto.cardenas@unad.edu.co https:// orcid.org/0000-0001-6263-3725 Universidad Nacional Abierta y a Distancia (UNAD). Colombia

\section{Repositorio de Arquitectura Empresarial del Gobierno (GEAR). Un Estado abierto, soportado en tecnologías de la información y la comunicación (TIC)}

\author{
Government Business Architecture \\ Repository (GEAR). An open state, \\ supported by information and \\ communication technologies (TIC)
}

\author{
Repositório de Arquitetura de Negócios \\ do Governo (GEAR). Estado aberto, \\ suportado pelas tecnologias da \\ informação e comunicação (TIC)
}

\begin{abstract}
Resumen
En el presente artículo se aborda el trabajo de investigación diseño e implementación de modelos orientados hacia el máximo aprovechamiento de las tecnologías de la información y la comunicación (TIC) en el contexto de la construcción de un estado abierto, eficiente, transparente, y participativo, que permita la prestación de servicios colaborativos para la comunidad.

Estos modelos se diseñan de acuerdo con los lineamientos de la administración pública, en términos del artículo 39 de la Ley 489 de 1998 y enmarcadas en el Plan Nacional de Desarrollo (PND) y los programas transversales de la gestión pública. Para este caso se trata de profundizar en el campo de la arquitectura empresarial (AE) como uno de los instrumentos que posibilita la puesta en marcha de proyectos soportados en TIC en Colombia.
\end{abstract}

Se considera la arquitectura empresarial (AE) como uno de los principales elementos estratégicos, cuyo fundamento teórico y operativo hace parte del análisis integral de las entidades gubernamentales, desde diferentes perspectivas y dimensiones, estas miradas críticas de las instituciones constituyen el insumo para poder realizar diagnósticos - pronósticos capaces de generar propuestas y alternativas que contribuyan a la transformación y modernización de las instituciones con base en el uso y la apropiación de tecnologías de la información y la comunicación (TIC).

Para el logro de los objetivos de implementación de las TIC y su aplicación en el campo de administración y la gobernanza, es fundamental partir del Marco de Referencia de AE para la gestión de tecnologías, aquí se trata de establecer un modelo de referencia para ser implementado como estructura orientadora de la arquitectura empresarial (AE). El 
marco de referencia o marco teórico, sobre la base de la estructura conceptual, consiste en la definición de los lineamientos sobre las prácticas significativas (memorables, significativas), que se han realizado con base en la utilización e implementación de las $\mathrm{AE}$ en el contexto de las tecnologías de la información y la comunicación (TIC).

El Repositorio de Arquitectura Empresarial del Gobierno (GEAR), se ha destacado por ser una de las estrategias de gobernabilidad en línea; este se soporta legalmente en el artículo 209 de la Constitución Política, el $3^{\circ}$ de la Ley 489 de 1998 y $3^{\circ}$ de la Ley 1437 de 2011, que establece los parámetros para la prestación de servicio de alta calidad, sobre la estrategia de la reutilización de datos públicos, su tratamiento y estandarización, lo mismo que su neutralidad, innovación y colaboración. Los fundamentos de la estrategia son desarrollados a partir de 4 componentes que facilitarán la masificación de la oferta y la demanda de Gobierno en línea. Estos son, TIC para servicios, TIC para el Gobierno abierto, TIC para la gestión y seguridad y privacidad de la información.

Palabras clave: análisis, arquitectura, datos, sistematización, gobernaza, tecnologías de la información.

\section{Abstract}

This article refers to the research work, design and implementation of models oriented towards the maximum use of Information and Communication Technologies (ICT) in the context of the construction of an open, efficient, transparent and participative state, that allows the provision of collaborative services for the community. These models are designed according to the guidelines of the public administration, in terms of article 39 of law 489 of 1998 and framed in the national development plan (PND) and the cross-cutting programs of public management. In this case, it is a question of going deeper into the field of Business Architecture $(\mathrm{AE})$ as one of the instruments that enables the implementation of ICT supported projects in Colombia.

$\mathrm{AE}$ Business Architecture is considered as one of the main strategic elements, whose theoretical and operational foundation is part of the comprehensive analysis of government entities, from different perspectives and dimensions, these critical views of the institutions are the input to carry out diagnoses - prognoses capable of generating proposals and Alternatives that contribute to the transformation and modernization of institutions based on the use and appropriation of ICT information and communication technologies.

In order to achieve the objectives of ICT implementation and its application in the field of administration and governance, it is essential to start from the AE Reference Framework for technology management, here it is about establishing a reference model to be implemented as a guiding structure for the $A E$ business architecture. The frame of reference or theoretical framework, based on the conceptual structure, consists of defining the guidelines on significant practices (memorable, significant), which have been made based on the use and implementation of EA in the context of ICT Information and Communication Technologies.

The Government Business Architecture Repository (GEAR), has stood out for being one of the online governance strategies, it is legally supported based on article 209 of the Political Constitution, the 3rd of Law 489 of 
1998 and 3 of Law 1437 of 2011, which establishes the parameters for the provision of high-quality service, on the strategy of reusing public data, its treatment and standardization, as well as its neutrality, innovation and collaboration. The foundations of the strategy are developed through 4 components that will facilitate the massification of supply and demand for online government. These are ICT for services, ICT for open government, ICT for information management and security and privacy.

Keywords: analysis, data, systematization, governance, information technologies.

\section{Resumo}

Este artigo trata do trabalho de pesquisa Design e implementação de modelos orientados para o uso máximo das tecnologias da informação e comunicação (TIC) no contexto da construção de um estado aberto, eficiente, transparente e participativo, isso permite a prestação de serviços colaborativos para a comunidade.

Esses modelos são elaborados de acordo com as diretrizes da administração pública, nos termos do artigo 39 da Lei 489 de 1998 e enquadrados no Plano Nacional de Desenvolvimento (PND) e nos programas transversais de gestão pública. Nesse caso, trata-se de aprofundar o campo da arquitetura de negócios (EA) como um dos instrumentos que possibilita a implementação de projetos apoiados pelas TIC na Colômbia.

A arquitetura de negócios (EA) é considerada um dos principais elementos estratégicos, cuja fundamentação teórica e operacional faz parte da análise abrangente das entidades governamentais, sob diferentes perspectivas e dimensões, essas visões críticas das instituições são a entrada para a realização de diagnósticos - previsões capazes de gerar propostas e alternativas que contribuam para a transformação e modernização das instituições com base no uso e apropriação das tecnologias da informação e comunicação (TIC).

Para alcançar os objetivos da implementação de TIC e sua aplicação no campo da administração e governança, é essencial começar a partir do Quadro de Referência de EA para gerenciamento de tecnologia, aqui é uma questão de estabelecer um modelo de referência a ser implementado como uma estrutura orientadora para arquitetura de negócios (AE). 0 quadro de referência ou referencial teórico, com base na estrutura conceitual, consiste em definir as diretrizes sobre práticas significativas (memoráveis, significativas), que foram feitas com base no uso e implementação da EA no contexto tecnologias da informação e comunicação (TIC).

O GEAR (Government Business Architecture Repository) se destacou por ser uma das estratégias de governança online; Isso é legalmente apoiado no artigo 209 da Constituição Política, 3 da Lei 489 de 1998 e 3 da Lei 1437 de 2011, que estabelece os parâmetros para a prestação de serviços de alta qualidade, sobre a estratégia de reutilização dados públicos, seu tratamento e padronização, bem como sua neutralidade, inovação e colaboração. Os fundamentos da estratégia são desenvolvidos a partir de 4 componentes que facilitarão a oferta e a demanda em massa do governo online. São TIC para serviços, TIC para governo aberto, TIC para gerenciamento de informações e segurança e privacidade.

Palavras-chave: análise, arquitetura, dados, sistematização, governança, tecnologia da informação. 


\section{INTRODUCCIÓN}

Con el avance de las tecnologías de la información y la comunicación (TIC), se ha ampliado notablemente la circulación de información a través de Internet, esta información de baja, mediana y alta complejidad, forma parte de una macrotendencia llamada en su momento la sociedad de la información; como una de las expresiones orientadas hacia la consolidación de la globalización moderna, la cual ha ganado presencia en casi la totalidad del planeta, en donde es empleado como parte de la construcción del contexto socioeconómico, político y cultural [1]. En un estudio elaborado con el propósito de documentar los avances europeos se señala que "las sociedades de la información se caracterizan por basarse en el conocimiento y en los esfuerzos por convertir la información en conocimiento". Cuanto mayor es la cantidad de información generada por una sociedad, mayor es la necesidad de convertirla en conocimiento.

La sociedad de la información y la información al conocimiento, ha generado grandes cambios en la naturaleza de las sociedades y en particular en el sentido crítico de la gobernabilidad y la nitidez en el orden administrativo, en el sentido de poder centrar su esfuerzo en la consolidación de una cultura global frente a la automatización y análisis de la información, orientada al desarrollo de las organizaciones y la producción de nuevos desarrollos que permiten afianzar los modelos económicos y generar transformaciones en las organizaciones con miras a consolidarlas en el marco mismo de la globalidad como ejemplo se tomaría a Europa, donde el término se masificó para el contexto de la Unión Europea [2].

El proyecto de incorporación de GEAR orientado para el establecimiento de un modelo de Gobierno abierto, a partir de la ejecución de una plataforma soportada en arquitectura de repositorios y estructurada en tecnologías de la información, se ampara y se regula legalmente bajo la reglamentación del Ministerio de Tecnologías de la Información y las Comunicaciones (MinTIC), Decretos 2573 de 2014, el cual define los lineamientos, instrumentos y plazos de la estrategia de Gobierno en línea para garantizar el máximo aprovechamiento de las TIC, con el fin de contribuir con la construcción de un estado abierto, eficiente, transparente, participativo y que preste mejores servicios con la colaboración de toda la sociedad [3].

Así mismo define el marco de referencia de arquitectura empresarial $(A E)$ para la gestión de tecnologías de la información: como un modelo de referencia puesto a disposición de las instituciones del Estado colombiano para ser utilizado como orientador transcendental de las arquitecturas empresariales, tanto sectoriales como institucionales. El marco establece la estructura conceptual, define lineamientos, incorpora mejores prácticas y orienta la implementación para lograr una administración pública más eficiente, coordinada y transparente, mediante el fortalecimiento de la gestión de las tecnologías de la información. El Decreto 1078 de 2015, por medio del cual se expide el Decreto Único Reglamentario del Sector de Tecnologías de la Información y las Comunicaciones. (Última actualización 25-04-2018), establece los objetivos y funciones que se encuentran definidas en la Ley 1341 de 2009, "por la cual se definen principios y conceptos sobre la sociedad de la información y la organización de las tecnologías de la información y las comunicaciones (TIC), se crea la Agencia Nacional de Espectro y se dictan otras disposiciones". y el decreto 415 de 2016, "por el cual se adiciona el Decreto único reglamentario del sector de la Función Pública, Decreto 1083 de 2015, en lo relacionado con la definición de lineamientos para el fortalecimiento institucional en materia de tecnologías de la información y las comunicaciones"; el cual exhorta a las entidades territoriales a adoptar lineamientos que las entidades estatales deben tener en cuenta para el fortalecimiento institucional y ejecución de los planes, programas y proyectos de tecnologías y sistemas de información en la respectiva entidad [4].Esta fundamentación legal se complementa con los dominios establecidos por el mismo MinTIC, a saber:

- Estrategia de TI.

- Gobierno de TI.

- Uso y apropiación.

- Información.

- Sistemas de información.

- Servicios Tecnológicos.

El Repositorio de Arquitectura Empresarial del Gobierno (GEAR), ha generado una serie de guías de implementación que se han delineado, para orientar a las entidades públicas en la implementación de la arquitectura empresarial, para la gestión de tecnologías. Esta metodología ha sido creada y validada por la alianza Business Support - UNAD, para la iniciativa del Departamento Administrativo de Ciencia, Tecnología e Innovación Colciencias, lo cual le da validez y seguridad a la propuesta. La primera parte de las guías busca generar un 
diagnóstico detallado sobre el estado del arte en el que se encuentra el modelo de gestión de una determinada entidad estatal. A partir de ese diagnóstico se definen las estrategias orientadas al mejoramiento de la eficacia, la competitividad y transparencia de la entidad desde la perspectiva y la estrategia de administración del Gobierno nacional [5], [6] y [7].

Para establecer una articulación entre el concepto de arquitectura empresarial y la implementación de GEAR, se establecen las siguientes fases:

Fase de diagnóstico inicial (AS-IS): el propósito de esta fase es el de definir el estado actual de la entidad, por cada dominio del marco de referencia de AE del Gobierno colombiano. AS-IS de Gobierno de TI, AS-IS de Estrategia de TI, AS-IS de uso y apropiación, AS-IS de información, AS-IS de sistemas de información y AS-IS de servicios tecnológicos. Esta fase se compone de los siguientes elementos:

- Instrumento de medición de avance FURAG.

- Elaboración de encuestas.

- Diagnóstico ligero.

- Diagnóstico detallado.

- Modelo de madurez AE.

- Gestión documental de artefactos de AE.

Fase de proyección futura o esperada (TO-BE): el propósito de esta fase es el de crear un estado ideal o futuro que la entidad de manera realista piense alcanzar como objetivo de su evolución. Esta fase se compone de los siguientes elementos:

- Road-Map y análisis de brechas.

- Modelo de madurez AE.

- Análisis de mejora AE.

- Modelo AHP de decisión.

- Gestión documental de artefactos de AE.

- Indicadores de gestión KPIs.

Fase de Road-Map y análisis de brechas: el propósito de esta fase es validar que la proyección esperada es realista, ver qué elementos de los 6 dominios van a ser evolucionados en cada uno de ellos y qué estrategias se deberían tomar para identificar las brechas entre el AS-IS y el TO-BE. Esta fase se compone de los siguientes elementos:
- Road-Map y análisis de brechas.

- Diagnóstico ligero.

- Diagnóstico detallado.

- Modelo de madurez AE.

- Control de la metodología de AE.

- Modelo AHP de decisión.

- Gestión documental de artefactos de AE.

Fase de portafolio de proyectos $\mathrm{AE}$ : el propósito de esta fase es crear formalmente el conjunto de decisiones identificadas en el análisis de brecha, con el fin de que todas las acciones de una iniciativa relacionada sean documentadas en un proyecto que la entidad debe formalizar públicamente conforme la ley colombiana [8], [9] y [10]. Las agrupaciones de todos estos proyectos se componen en el portafolio de proyectos y serán los que permitan el desarrollo de la arquitectura de solución. Esta fase se compone de los siguientes elementos:

- Road-Map y análisis de brechas.

- Modelo de madurez AE.

- Control de la metodología de AE.

- Gestión de proyectos e iteraciones de AE.

- Modelo AHP de decisión

- Gestión documental de artefactos de AE.

- Mitigación del riesgo.

Fase de ejecución, control y seguimiento del portafolio: el propósito de esta fase consiste en crear las arquitecturas de transición y realizar el control sobre los artefactos de AE que están evolucionando, el control de los proyectos y en general el seguimiento de qué tanto se está cerrando la brecha entre el AS/IS y el TO/BE. Esta fase se compone de los siguientes elementos:

- Road-Map y análisis de brechas.

- Modelo de madurez AE.

- Control de la metodología de AE.

- Gestión de proyectos e iteraciones de AE.

- Modelo AHP de decisión.

- Gestión documental de artefactos de AE.

- Gestión de hojas de vida: clientes, procesos, macroprocesos, Sistemas de información, productos, servicios, protección de datos. 


\section{Los pasos de un proyecto de Arquitectura Empresarial}

Un proyecto de esta índole requiere de un proceso de planeación estratégica cuyo propósito es ordenar la organización y transformarla hacia un estado deseado que siempre apunta hacia el cumplimiento de la misión y la estrategia general. Dada la magnitud y el alcance de un proyecto de Arquitectura Empresarial, es lógico que no se trata de un proyecto que deba impulsar el $\mathrm{CIO}$ o su área de $\mathrm{TI}$, sino que debe tener el respaldo total de la alta gerencia desde su propio comienzo. Esto no siempre resulta fácil para los $\mathrm{ClO}$, pues aún sus áreas son vistas como simple soporte de la operación, como algo secundario. Por ello, los $\mathrm{ClO}$ deben ser más estratégicos y tener mayor capacidad de interlocución con los líderes de sus organizaciones. Y como se trata de todo un desafio, especialmente para los $\mathrm{CIO}$ públicos, el Ministerio TIC les brinda apoyo en formación, con eventos como el $\mathrm{ClO}$ Summit y con otros recursos y herramientas. Las palabras del Ministro Diego Molano Vega durante la apertura del Cio Summit no fueron casualidad: "Cuenten con nosotros para ayudar a convencer a sus jefes y equipos directivos para la implementación de TI".

Ya con el apoyo de los directivos de la organización o entidad, se inicia un proceso de planeación de la Arquitectura Empresarial con los siguientes niveles:

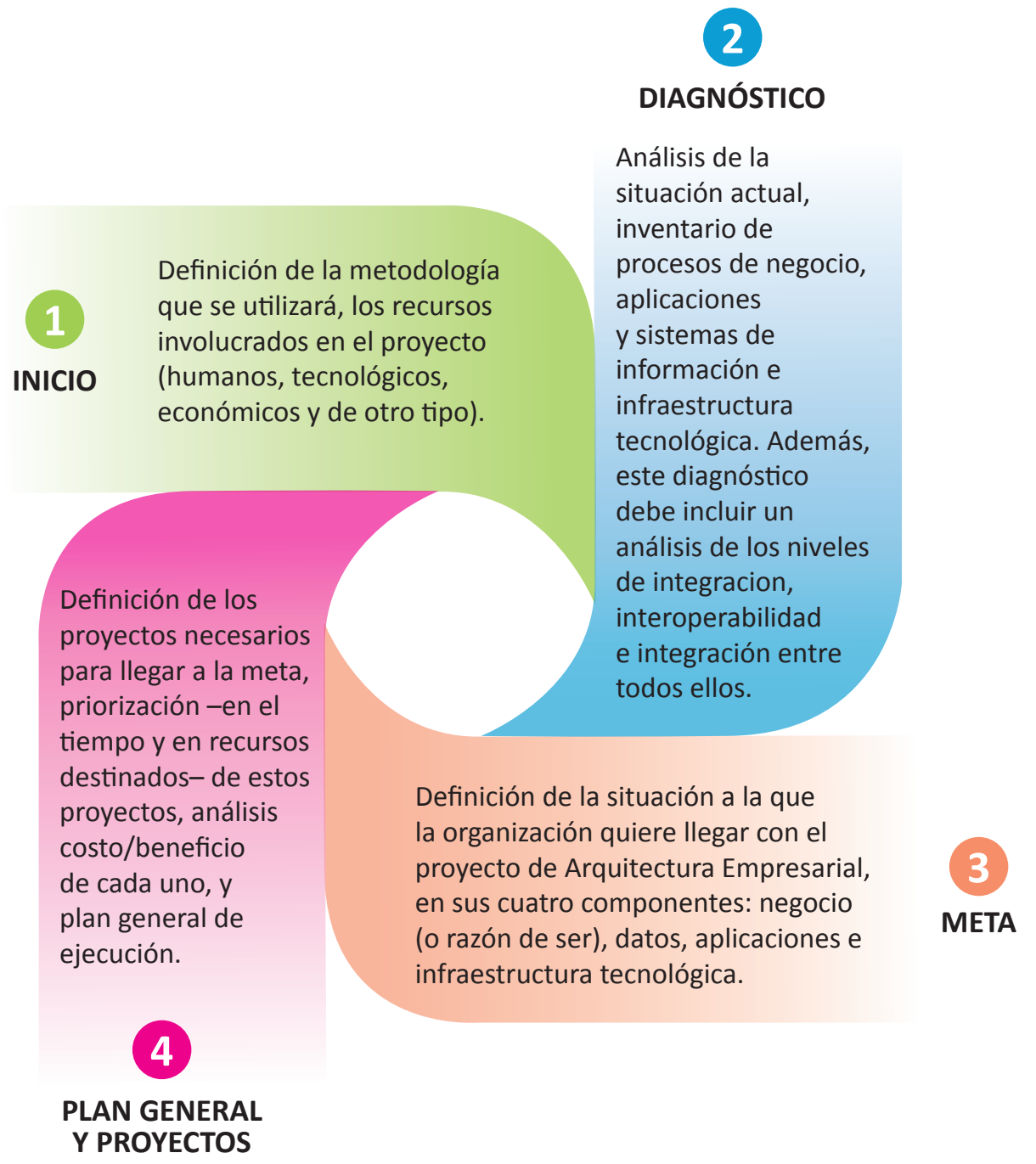

Figura 1. Pasos de un proyecto de arquitectura empresarial.

Fuente: [11]. 


\section{EL PROBLEMA}

El Gobierno nacional, a través del MinTIC ha definido reglas de alto nivel en Arquitectura de TI para las entidades del Estado. Esta reglamentación tiene como fin lograr que cada entidad pública del país tenga estrategias claras sobre cómo debe usar sus recursos tecnológicos, con el fin de que estas, sean un apoyo concreto en el desarrollo de las actividades de las entidades públicas.

Una de las principales herramientas con las que cuenta una organización para gestionar y alinear el recurso de tecnologías de la información (TI) desde su perspectiva estratégica es la arquitectura empresarial $(\mathrm{AE})$. En referencia a la Estrategia de Gobierno en Línea (GEL), la implementación de una arquitectura al interior de las organizaciones gubernamentales se perfila como el principal instrumento para efectuar la arquitectura $\mathrm{TI}$ en Colombia y habilitar las estrategias de TIC para servicios, TIC para la gestión, TIC para el Gobierno abierto y para la seguridad y la privacidad.

Sin embargo, son muchas las organizaciones que aún no han tenido un primer acercamiento, ni con la estrategia de gobierno en línea, ni con las herramientas de $A E$ para su implementación y evolución. Por tanto, esta fase de desarrollo de un diagnóstico detallado busca definir una línea base para la instauración de un ejercicio de arquitectura empresarial en una entidad gubernamental, que permita dar inicio a esta estrategia y obtener un diagnóstico preliminar de la organización [12], [13] y [14].

Grupos de interés y sus preocupaciones: para poder realizar una AE efectiva, se requiere de los siguientes grupos de interés:

- Departamento de TI de la organización.

- Representantes de los entes administrativos y de toma de decisiones.

- Departamento de planeación.

- Comité de AE.

- Lista de problemas / escenarios a abordar.

No existe un acercamiento previo a la estrategia de gobierno en línea y no se tiene conocimiento de arquitecturas empresariales como herramienta de gestión en la organización [15].

No existe un acercamiento previo a la estrategia de gobierno en línea, pero se tiene conocimiento de arquitecturas empresariales como herramienta de gestión y se han realizado algunas aproximaciones.
Existe un acercamiento previo a la estrategia de gobierno en línea, se tiene conocimiento de arquitecturas empresariales como herramienta de gestión y se han realizado algunas aproximaciones.

Los recursos económicos de los municipios y entidades nacionales no son iguales, por lo que GEAR, busca facilitar su acceso por medio de planes acordes con el tamaño, presupuesto y necesidades de la entidad participante. MINTIC 2018

\section{ALCANCE DEL PROYECTO}

GEAR es una herramienta cuyo foco es servir como repositorio de arquitectura empresarial con una interfaz gráfica amigable en la Web y que puede ser expuesta en la nube para que las entidades no tengan que preocuparse por el montaje y mantenimiento de infraestructura del software [16].

GEAR en su estructura tendrá simplicidad para el manejo de los procesos y será una herramienta "que guie" al usuario y que le facilité el proceso de cumplimiento de la regulación colombiana y el alcance de los objetivos de esta.

GEAR será una herramienta que proporcioné al usuario el cómo se implementan los lineamientos de alto nivel establecidos por el MinTIC para cumplir la reglamentación colombiana y el alcance de los objetivos propuestos.

\section{USO Y APROPIACIÓN DE ARQUITECTURA TI}

Uso y apropiación de las tecnologías son conceptos que se asocian a todos los campos de la sociedad, y en particular al campo de la gestión administrativa y la gestión pública; aquí es importante recalcar que uno de los obstáculos que se presenta precisamente en la aplicabilidad de las TI, es el de la apropiación, puesto que establece una relación directa con la cultura y los comportamientos culturales de las personas y de los usuarios de las TI. En este sentido las instituciones han generado una serie de espacios orientados hacia la sensibilización a las tecnologías, esto implica el desarrollo de programas de formación y actualización orientados desde las necesidades de las poblaciones y desde el mismo manejo de la información y su posterior utilización por parte de los usuarios [17]. Este aspecto que para unos se convierte en simple capacitación, para otros es precisamente la profundización en la cultura para el uso de la tecnología, implica además conocer precisamente en el sentido crítico, 
por qué usamos las TI y cuáles son los impactos de ellas en las poblaciones objeto de los proyectos soportados con tecnologías de la información. Se deben tomar como referencia varios aspectos que posibiliten esa apropiación y ese acercamiento a la cultura tecnológica, entre otros:

\section{A. Usabilidad}

Como la condición que deben tener los contenidos que van a ser abordados por los usuarios y por el público en general, información pertinente y apropiada de acuerdo con las necesidades de las personas y sus problemáticas por resolver; implica también que la información pueda ser de libre acceso a todos los públicos y que sea lo más completa posible.

\section{B. Navegabilidad}

Como la condición que deben tener las presentaciones de los contenidos y que permiten precisamente que los usuarios puedan ingresar y buscar de manera clara y sin confusiones, la información correspondiente a sus preguntas y a sus necesidades, esto implica una muy clara, transparente y limpia entrega de los contenidos, una buena disponibilidad y un muy buen sistema de información asociada a los contactos y ampliación de la información, esto implica que los contenidos digitales puedan ser usados y aplicados a la resolución de problemas y preguntas de los usuarios.

\section{Accesibilidad}

Tiene relación directa con los mínimos de tecnología y distribución, esto implica una red permanentemente abierta con unas condiciones técnicas en las que la rapidez y la velocidad estén asociadas a las categorías de la información, audios, videos, textos, animaciones, etc. Así mismo la condición de ingreso a todos los públicos de manera fácil y fluida.

\section{Disponibilidad}

En la arquitectura empresarial es vital la realización de estudios sobre la disposición de la infraestructura que permitirá el acceso a la información. Algunas empresas, sobre todo en el sector de la banca y del comercio han incorporado el concepto de los kioscos digitales, como una manera de ampliar los espacios físicos de las empresas y poder socializar la información a través de estos dispositivos, recientemente, se relaciona el concepto de banca móvil, por ejemplo, orientado a la realización de transacciones rápidas, que inclusive abarca el sector internacional [15].

\section{E. Actualización y sostenibilidad}

En lo relacionado a la capacidad de las tecnologías y su acondicionamiento a las necesidades de los sectores empresariales, implica actualizaciones en software y hardware, requiere una actualización de los contenidos que se ofrecen, reseñados anteriormente, y de la infraestructura, que como ya sabemos, cambia permanentemente y a velocidades imprevisibles.

\section{F. Desarrollo de proyectos}

Incluye aquí la condición de superar el concepto de la compra de tecnología sin hacer las previsiones del caso y sin una previa elaboración de estudios para su adopción. Aquí el criterio de "si la competencia" implementa tal o cual tecnología, la empresa debe adquirir la misma para "no quedarse atrás", debe ser superado mediante asesorías y orientaciones de personas capacitadas en el tema, con base en el desarrollo de proyectos de evaluación para la adopción de tecnologías. Estos proyectos permiten generar los espacios, las condiciones, los elementos genéricos que contribuyan a la consolidación de unas buenas prácticas con uso de arquitectura..

\section{G. Evaluación}

Es necesario disponer de los espacios para realizar evaluaciones permanentes sobre la implementación de los proyectos, evaluaciones que van desde las condiciones de prestación de servicios, hasta el acompañamiento al usuario y su posterior satisfacción. Aquí es importante, no solo la calificación del servicio (B-R-M), sino más bien tener el conocimiento necesario para poder solucionar las posibles fallas que presente el proyecto. El sistema de evolución desde la perspectiva de conocer cuál es la situación real y cuál es el escenario adecuado, en el sentido de los objetivos y las metas trazados en el proyecto. En general valorar el nivel de satisfacción del usuario en el uso de la arquitectura TI, así mismo la valoración a partir de herramientas 
básicas, analíticas, y gerenciales, que se orienten a la comunicación, la divulgación, la realimentación, y la gobernanza en el uso y la apropiación de arquitectura $\mathrm{Tl}$.

Lo anterior forma parte de la estructura organizacional y operativa para la implementación de proyectos $\mathrm{TI}$ - y proyectos GEAR, generada desde el estado colombiano y obedeciendo a los principios de modernización y transparencia en torno a los objetivos de desarrollo y el alcance de las metas planteadas por el Gobierno actual. Se entiende que estos proyectos deben contar con espacios para la formación del capital humano que se desempeña en el campo de la gestión pública. Esto implica seguramente la puesta en marcha de proyectos asociados a la actualización de los funcionarios en el uso y la apropiación de las tecnologías y comprender el alcance de los proyectos y sus implicaciones en los procesos de desempeño de funciones de cada uno de los funcionarios: "los funcionarios públicos deben conocer, entender y establecer las brechas entre la situación actual y la situación deseada, además de tener las capacidades requeridas para actuar en la transformación de sus entidades, sus municipios, departamentos y el Estado en su totalidad” [18].

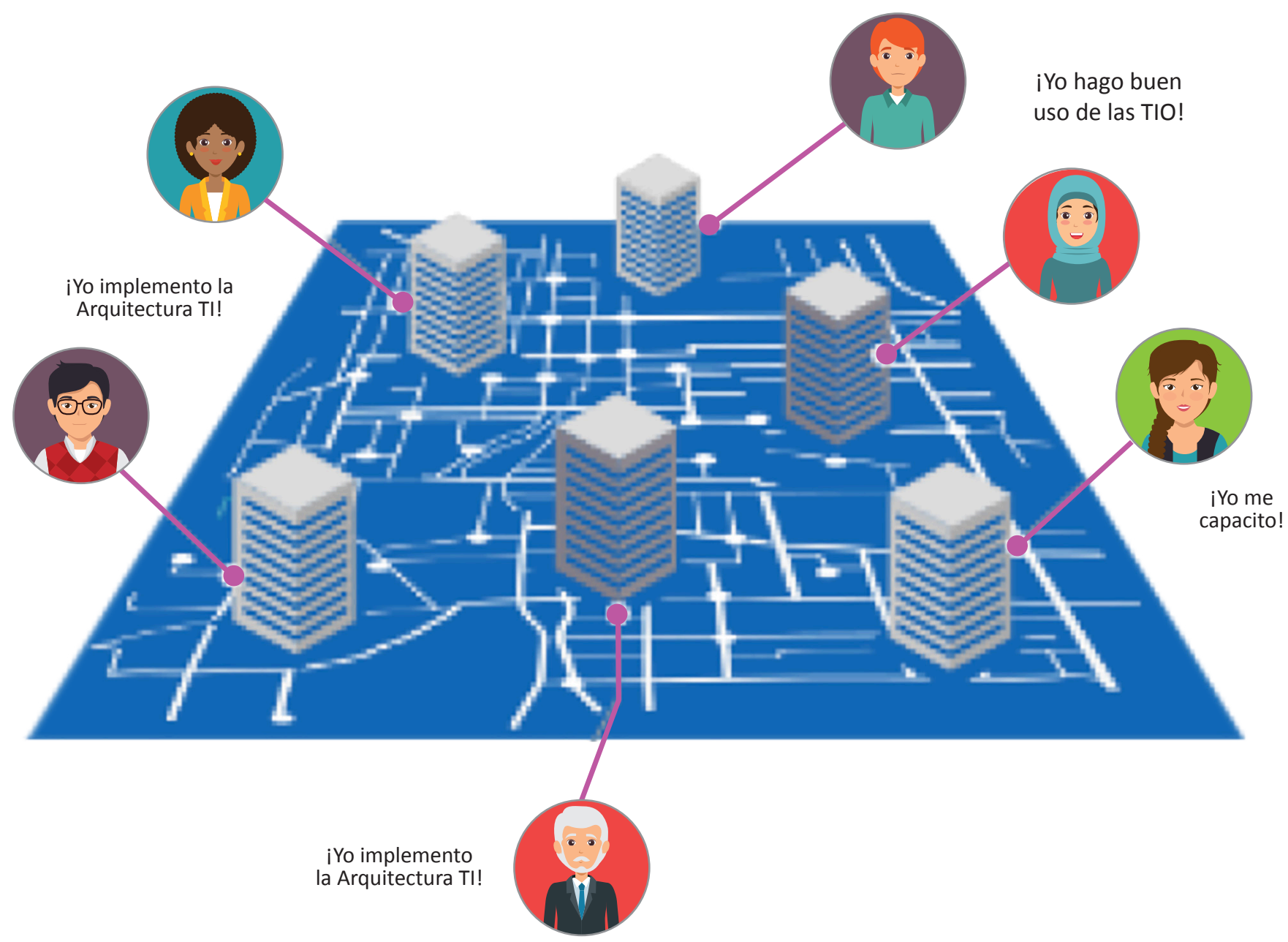

Figura 2. Uso y apropiación de Arquitectura TI. Fuente: [19]. 
Con la implementación y el desarrollo de la arquitectura TI y los Repositorio de Arquitectura Empresarial del Gobierno (GEAR), el Estado debe alcanzar unos niveles que permitan la conformación de un capital humano altamente cualificado y con alta capacidad de resolución de las problemáticas actuales, relacionadas con el manejo efectivo de la información y de la comunicación entre los usuarios (ciudadanos en general) y las instituciones gubernamentales, aquí uno de los propósitos fundamentales del Estado, es mejorar la comunicación entre las instituciones y la sociedad en su conjunto, para reconocer los avances en los planes, programas y proyectos de los gobiernos, esto implica profundizar en la eficiencia, transparencia y operabilidad de las instituciones con la participación activa de las comunidades.

\section{BENEFICIOS Y RETOS}

Con la implementación de la arquitectura TI se pueden establecer las condiciones para que se puedan generar espacios de comunicabilidad, entendida como la relación entre la información, el proveedor y la comprensión de esta información por parte de los usuarios (ciudadanos en general). Estos grados de comunicación son posibles a partir de la incorporación de modelos como por ejemplo los repositorios de arquitectura empresarial, que son uno de los recursos de la $\mathrm{TI}$, para almacenar y proveer la información pertinente y apropiada, que cumpla con las condiciones y las necesidades de los ciudadanos. Esto implica un alto compromiso por parte de los funcionarios del Estado en el desarrollo de aprendizajes y formación en el uso y en la apropiación de TI. Cobra sentido este aspecto, en la medida en que permita que todos y cada uno de los funcionarios se involucren de manera directa en la definición de estas mismas arquitecturas en las empresas sectoriales, territoriales y nacionales del Estado. En este sentido se cuenta precisamente con el conocimiento y las habilidades de estas comunidades que hacen posible la construcción y la conformación de verdaderos repositorios de información que se asocian a la arquitectura $\mathrm{TI}$ [20].

Este aspecto es la base de los proyectos TI, el capital humano actualizado y con altos niveles de apropiación permiten la conformación de líderes de los sectores y de los territorios que conformen y desarrollen comunidades de aprendizaje participando activamente en la elaboración y desarrollo de proyectos de arquitectura $\mathrm{TI}$, en este orden es posible generar una transformación cultural y conceptual que permita tener otra mirada y nuevos escenarios que faciliten la adopción de tecnologías de la información, así mismo que en las posteriores incorporaciones de tecnología, el impacto debe ser menor, ya que se cuenta con estas comunidades de práctica de favorecen el avance y la consolidación de los proyectos relacionados a la arquitectura de TI, y en general con todos los proyectos asociados al uso y apropiación de $\mathrm{TI}$ en los diferentes niveles de gobierno [21].

De otro lado, se contempla que en la medida en que se establezca el cierre de las brechas en lo relacionado con la información y la comunicación, se establece el criterio de la transparencia de los proyectos de desarrollo y de inversión, en ese sentido se logra la optimización de los recursos económicos, humanos y técnicos, ya que con el recurso de una arquitectura empresarial TI, no solo se logra la eficiencia y el manejo de una información pertinente y apropiada para el ciudadano y toda la comunidad, sino también que reduce enormemente los costos operativos de los proyectos, puesto que sus procesos y ejecuciones deben ser planeadas y alineadas desde la perspectiva de las estrategias y los propósitos de las entidades y de los sectores involucrados, todo ello impactará de manera directa en una mejora de servicios que prestan las empresas tanto privadas como estatales, que lleva altos niveles de gobernabilidad y gobernanza. Con ello se logra:

- Desarrollar una arquitectura empresarial institucional cuyo gran objetivo sea ofrecer servicios más eficientes a los ciudadanos y que además se encuentre alineada con las arquitecturas sectoriales de su territorio.

- Permitir que todos los proyectos de TI de las entidades estén alineados con la estrategia definida por el territorio.

- Lograr que la institución funcione como un todo, con un norte estratégico, claro, que le permita ofrecer servicios eficientes y oportunos [18], [22], [23] y [24]. 


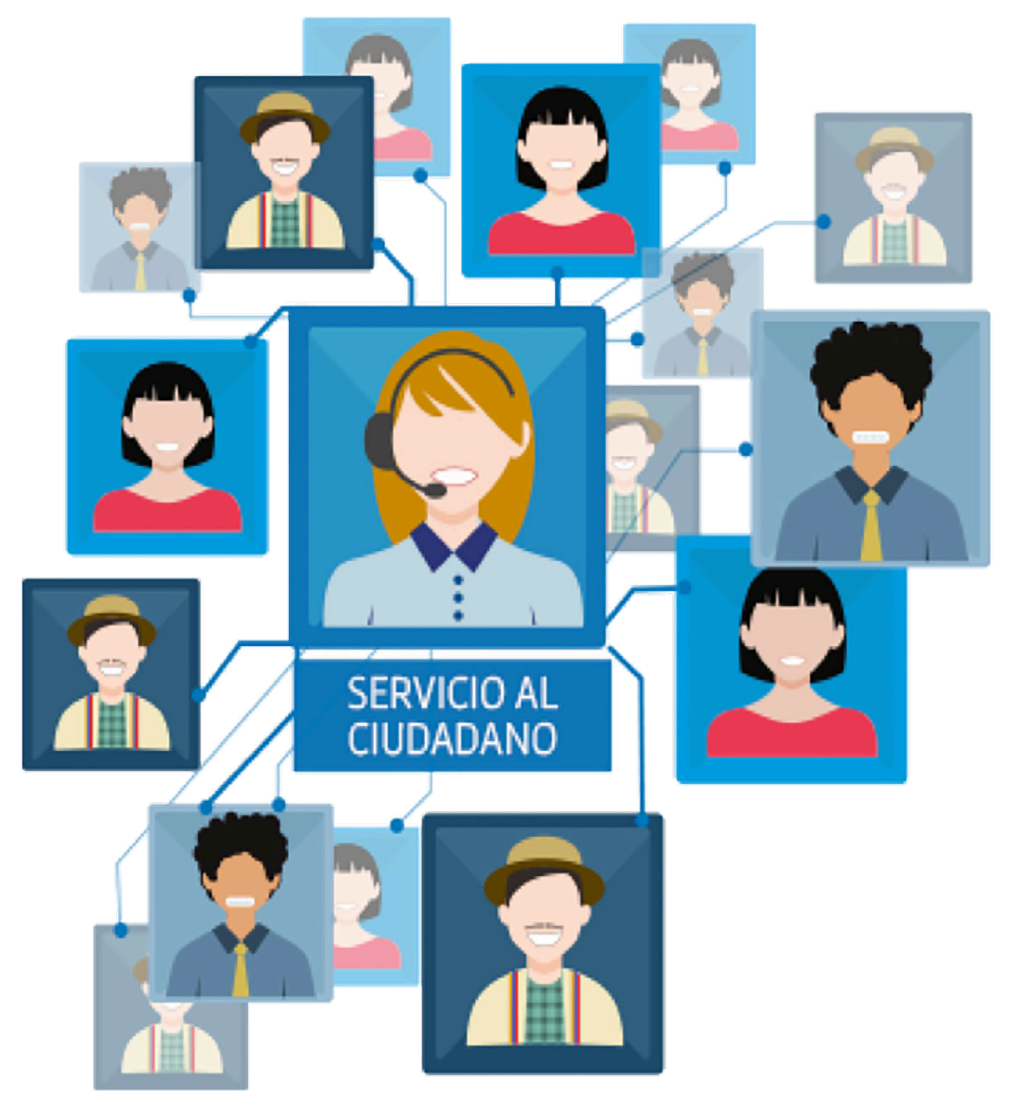

Fig. 3. Servicio al ciudadano.

Fuente: [24].

\section{REFERENCIAS}

[1] R. Trejo Delarbre, La nueva alfombra mágica. Usos y mitos de Internet, la red de redes. $1^{\text {a }}$ ed., México: Fundesco, 1996.

[2] R. M. Roberto Mauricio, "Big Data el nuevo orden de la información y la comunicación", Publ. investig., vol. 13, n. ${ }^{\circ} 2$, pp. 93-99, jul. 2019.

[3] S. M. Arango y Londoño, "Enterprice architecture: a general scope", Revista Ingenierías Universidad de Medellin, vol. 9, p. 16, 2010.

[4] MinTIC. (2016). "Decreto 415 de 07 de marzo de 2016". [En línea]. Disponible en: https:// www.mintic.gov.co/portal/604/w3-article-61527.html.
[5] D. Brown y P. Bahrs, "Arquitectura empresarial para Ingenieros de Sistemas", [En línea]. Disponible en: https://www.ibm.com/developerworks/ssa/rational/library/edge/09/ jun09/enterprisearchitecture/index.html. [Último acceso: 0211 2018].

[6] R. Carnabal y A. Cabarcas, "Aplicacion de un esquema de Arquitectura Empresarial (Togaf) para una Pequeña empresa PYME utilizando aplicaciones colaborativas de Google", Información Tecnológica, vol. 28, pp. 85-92, 2017.

[7] B. H. Cruz y P. W. Briceño, “Identificación de principios de arquitectura empresarial para la gestión de factores de impacto en entidades públicas colombianas utilizando Togaf", Inventum Ingenieria, vol. 10, pp. 22-28, 2015. 
[8] H. Jonkerset, "Enterprise architecture: Management tool and blueprint for the organization", Information Systems Frontiers, vol. $8, \mathrm{n}^{\circ} 2$, pp. 63-66, 2006.

[9] S. Kaisleret, "Enterprise Architecting: Critical Problems", en 38th Hawaii International Conference on System Sciences, Hawaii, 2005, pp.

[10] M. Lankhorst, Enterprise Architecture at Work. Modeling, Communication and Analysis, Berlin: Springer, 2016.

[11] MinTIC. (2013, Diciembre 2). "Un Estado integrado: La meta de la arquitectura empresarial para Colombia”. Cio@gov. n² 2, pp. 4-9. [En línea]. Disponible en: https://www.mintic.gov.co/gestionti/615/articles-5322_Revista_pdf.pdf

[12] F. De Boeret, "Change Impact Analysis of Enterprise Architectures", en 2005 IEEE International Conference on Information Reuse and Integration, Las Vegas, 2005, pp. 15-17.

[13] F. Goethalset, "Managements andenterprice architecture click: The FADE framework", Information Systems Frontiers, vol. 8, $\mathrm{n}^{\circ} 2$, pp. 67-79, 2006.

[14] C. Granja, M. Vallejo, Adopción de un marco metodológico de arquitectura empresarial en una empresa gubernamental, Tesis de grado previa a la obtención del título de Magíster en Gerencia de Tecnologías de la Información, Escuela de Sistemas, Facultad de Ingeniería, Pontificia Universidad Católica de Ecuador, 2014.

[15] M. A. Malleuve, Alfonso y R. D., "Study of elements behavior for integration management system with enterprise architecture approach", Dyna, vol. 84, n²03, pp. 349355, 2017.
[16] R. J. Martelo, I. Blanquicet y L. Rodríguez, "Metodología para Seleccionar Aplicaciones de la Tecnología de Comunicaciones Voz sobre la IP (VolP) para pequeñas y medianas empresas (Pymes). El caso de una Agencia de Viajes, Información Tecnológica, Vol. 26, $\mathrm{n}^{\circ}$ 6, pp. 121-128, 2015.

[17] J. M. J. Miller, Model Driven Architecture, Technology Committee and Architecture Board, Editors: Joaquin Miller and Jishnu Mukerji. United States on December 13, 2010.

[18] MinTIC. 2018. "Ministerio de las Tecnologías de la Información y las Comunicaciones". [En línea]. Disponible en www.mintic.gov.co.

[19] MinTIC. "Uso y aplicación". [En línea]. Disponible en: https://mintic.gov.co/arquitecturati/630/w3-propertyvalue-8120.html.

[20] MinTIC. (2014). "Arquitectura empresarial. El camino hacia un gobierno integrado". [En línea]. Disponible en: http://www.mintic. gov.co/gestionti/615/articles-5322_Revista_pdf.pdf

[21] A. Molano. (2017). "Qué es arquitectura empresarial”. [En línea]. Disponible en: https: // repositorio.escuelaing.edu.co/bitstream/001/690/2/Var\%C3\%B3n\%20G.\%2C \%20Yenny\%20Paola\%20-\%202017.pdf [22] P. Project Management Institute, Fundamentos para la dirección de proyectos, $5^{\mathrm{a}}$ ed Ciudad: Newtown Square, Pensilvania 190733299 EE.UU. Springer-Verlag, pp.3 -58 2013.

[23] S. A. Sanin. (2005). "Definición de una arquitectura empresarial en una empresa que nace con un legado complejo". [En línea]. Disponible en: https://slideplayer.es/ slide/1700958/

[24] MinTIC. "Marco de Referencia de Arquitectura v. 2.0". [En línea]. Disponible en: https:// mintic.gov.co/arquitecturati/630/w3-propertyvalue-8118.html. 